\title{
Calphostin C-induced apoptosis is mediated by a tissue transglutaminase-dependent mechanism involving the DLK/JNK signaling pathway
}

\author{
K Robitaille ${ }^{1,2}$, A Daviau ${ }^{1,2}$, G Lachance ${ }^{1}$, J-P Couture ${ }^{1}$ and R Blouin ${ }^{\star, 1}$
}

A role for tissue transglutaminase (TG2) and its substrate dual leucine zipper-bearing kinase (DLK), an upstream component of the c-Jun N-terminal kinase (JNK) signaling pathway, has been previously suggested in the apoptotic response induced by calphostin $\mathrm{C}$. In the current study, we directly tested this hypothesis by examining via pharmacological and RNA-interference approaches whether inhibition of expression or activity of TG2, DLK and JNK in mouse NIH 3T3 fibroblasts and human MDA-MB231 breast cancer epithelial cells affects calphostin C-induced apoptosis. Our experiments with the selective JNK inhibitor SP600125 reveal that calphostin C is capable of causing JNK activation and JNK-dependent apoptosis in both cell lines. Small interfering RNA-mediated depletion of TG2 alone strongly reduces calphostin $\mathrm{C}$ action on JNK activity and apoptosis. Consistent with an active role for DLK in this cascade of event, cells deficient in DLK demonstrate a substantial delay of JNK activation and poly-ADP-ribose polymerase (PARP) cleavage in response to calphostin C, whereas overexpression of a recombinant DLK resistant to silencing, but sensitive to TG2-mediated oligomerization, reverses this effect. Importantly, combined depletion of TG2 and DLK further alters calphostin C effects on JNK activity, Bax translocation, caspase-3 activation, PARP cleavage and cell viability, demonstrating an obligatory role for TG2 and DLK in calphostin C-induced apoptosis.

Cell Death and Differentiation (2008) 15, 1522-1531; doi:10.1038/cdd.2008.77; published online 23 May 2008

Calphostin $\mathrm{C}$ is a fungus-derived antibiotic that has been identified as a potent and selective inhibitor of protein kinase $\mathrm{C} .{ }^{1} \mathrm{~A}$ number of studies have demonstrated that calphostin $\mathrm{C}$ can induce apoptosis in different cellular backgrounds $^{2,3}$ by mechanisms that are still largely unknown, but might include activation of caspase- $3,{ }^{4}$ Bax translocation to the mitochondria ${ }^{5}$ and inhibition of phospholipase D. ${ }^{6}$ Because calphostin $C$ has the ability to substantially increase intracellular $\mathrm{Ca}^{++}$level, ${ }^{3}$ it also rapidly and strongly switches on the crosslinking function of tissue transglutaminase (TG2) in various experimental systems. ${ }^{7-9}$ TG2 is a multifunctional enzyme with a transamidating activity and has a role as a signal-transducing GTP-binding protein. ${ }^{10,11}$ As a member of the transglutaminase family, TG2 exhibits a $\mathrm{Ca}^{2+}$-dependent covalent crosslinking activity that allows the formation of $\gamma$-glutamyl-e-lysine isopeptide bonds within or between polypeptide chains and also allows the incorporation of polyamines into substrate proteins. Such transglutaminasemediated crosslinking of substrate proteins has been proposed to regulate important biological processes, such as apoptosis. ${ }^{10-12}$ The role of TG2 in dying cells remains puzzling as it has been shown to promote or prevent apoptosis, depending on the cell context and the nature of the death stimulus. Recent studies from different laboratories have revealed that the positive or negative effects of TG2 on apoptosis are modulated by both its transamidating (e.g., protein crosslinking and polyamination) and non-transamidating (e.g., GTP binding/hydrolysis) functions. ${ }^{13-15}$ Activation of the transamidating function of TG2 in cells undergoing spontaneous or stress-induced apoptosis has been found to cause the polymerization of specific cytosolic, cytoskeletal and nuclear substrate proteins, such as the retinoblastoma protein, ${ }^{7}$ actin $^{16}$ and core histones, ${ }^{17}$ which are thought to contribute in some way to the early and late stages of the apoptotic process.

We have reported previously that calphostin C-mediated death of cells such as NIH 3T3 fibroblasts and Neuro-2a neuroblastomas is associated with the rapid and dramatic downregulation of dual leucine zipper-bearing kinase (DLK), a mitogen-activated protein kinase kinase kinase (MAPKKK) of the stress-activated c-Jun N-terminal kinase (JNK) signaling pathway. ${ }^{9}$ DLK downregulation in calphostin C-treated cells was found to be paralleled by the accumulation of high molecular weight DLK oligomers, the formation of which is almost completely antagonized by the transglutaminase inhibitor monodansylcadaverine. ${ }^{9}$ Treatment with monodansylcadaverine prior to stimulation with calphostin $C$ also blocked cleavage of the caspase substrate poly-ADP-ribose polymerase (PARP), suggesting that activation of TG2 is necessary to promote DLK oligomerization and to induce apoptosis. Interestingly, upon TG2-mediated oligomerization, ectopically expressed DLK was found to function as a

\footnotetext{
${ }^{1}$ Département de biologie, Faculté des sciences, Université de Sherbrooke, Sherbrooke, Québec, J1K 2R1, Canada

*Corresponding author: R Blouin, Département de biologie, Faculté des sciences, Université de Sherbrooke, 2500, boulevard de l'Université, Sherbrooke, Québec J1K 2R1, Canada.

Tel: + 8198218000 ext. 62062; Fax: + 819821 8049; E-mail: Richard.Blouin@ USherbrooke.ca

${ }^{2}$ These authors contributed equally to this work.

Keywords: calphostin C; apoptosis; tissue transglutaminase; dual leucine zipper-bearing kinase; c-Jun amino-terminal kinase

Abbreviations: TG2, tissue transglutaminase; DLK, dual leucine zipper-bearing kinase; JNK, c-Jun amino-terminal kinase; PARP, poly-ADP-ribose polymerase

Received 03.12.07; revised 22.4.08; accepted 28.4.08; Edited by M Piacentini; published online 23.5.08
} 
constitutively active protein kinase, which in turn stimulates the JNK signaling pathway and sensitizes cells to calphostin C-induced apoptosis. Taken as a whole, these observations clearly argue for a role of DLK polymerization and subsequent JNK activation in the molecular mechanisms by which TG2 regulates calphostin $\mathrm{C}$-induced apoptosis.

As a mean of exploring this notion further, we examined herein the contributory roles of endogenous TG2, DLK and JNK in calphostin C-induced apoptosis using pharmacological and RNA-interference approaches. Our results show that calphostin $\mathrm{C}$ causes apoptosis in mouse NIH 3T3 fibroblasts and human MDA-MB-231 breast cancer cells through activation of the JNK pathway. Knockdown of either TG2 or DLK significantly reduces calphostin $\mathrm{C}$ action on JNK activity and apoptosis, whereas combined depletion of both proteins attenuates this response even more. Furthermore, we show that TG2 knockdown blocks calphostin C-induced DLK oligomerization, confirming the crucial role played by TG2 in this response. Together, these data greatly extend our previous work done in cells with ectopic expression of TG2 and $\mathrm{DLK}^{9}$ and demonstrate for the first time that endogenous TG2, DLK and JNK are part of a sequential signaling pathway that mediates the effects of calphostin $C$ on apoptosis.

\section{Results}

Calphostin C-induced apoptosis in NIH 3 T3 cells is mediated by the JNK signaling pathway. A role for JNK in cell-signaling events that are pro-apoptotic is well established. ${ }^{18}$ In some cell types, such as in neurons, JNK has been recognized as an important regulator of cell death evoked by a variety of apoptotic stimuli, including excitotoxic stress, withdrawal of growth factors, DNA damage, oxidative stress and $\beta$-amyloid exposure. ${ }^{19-22}$ To investigate the relative contribution of JNK in calphostin C-induced apoptosis, we first examined by immunoblot analysis whether its phosphorylation on Thr-183 and Tyr-185, an indicator of activation, is induced in NIH 3T3 cells exposed to calphostin $C$ for various times. In agreement with our previous findings, ${ }^{8}$ a gradual and dramatic increase in JNK activity was detected as early as $45 \mathrm{~min}$ following treatment with $250 \mathrm{nM}$ calphostin C (Figure 1a), a dose known to decrease survival in several cell types. ${ }^{23,24}$ Concurrent immunoblotting of the lysates with an antibody insensitive to JNK phosphorylation state revealed that the total amount of JNK remained constant under these treatment conditions. Apoptosis was assessed in these cells by probing the lysates with antibodies against cleaved forms of caspase-3, one of the key effector molecules in apoptotic cell death, ${ }^{25}$ and the caspase substrate PARP. ${ }^{26}$ Results shown in Figure 1a indicate that calphostin $\mathrm{C}$ was able to stimulate the cleavage of caspase-3 as early as $75 \mathrm{~min}$ after initiation of the treatment whereas PARP cleavage occurred about $15 \mathrm{~min}$ earlier. To determine whether calphostin $\mathrm{C}$-induced cleavage of caspase-3 and PARP was indeed attributable to JNK activation, we next performed immunoblotting experiments with NIH 3T3 cells pretreated with the JNK inhibitor SP600125 27 prior to calphostin C treatment. As shown in Figure 1a, SP600125 completely prevented the calphostin
C-mediated accumulation of cleaved caspase-3 and PARP, thereby suggesting that JNK activation contributes to apoptosis in response to calphostin C.

As calphostin C- and JNK-mediated apoptosis was reported previously to be associated with the translocation of the proapoptotic protein Bax to mitochondria, ${ }^{5,28}$ a process essential for cytochrome $c$ release and induction of apoptosis, it was also of interest to determine whether calphostin $C$ treatment induces the mitochondrial redistribution of $\mathrm{Bax}$ in NIH 3T3 cells and whether JNK activity is required for such an event. To do so, NIH 3T3 cells were preincubated in the absence or in the presence of SP600125 for 60 min before being exposed to calphostin $\mathrm{C}$ and subjected to subcellular fractionation. As shown in Figure 1b, we found that treatment of NIH 3T3 cells with calphostin $C$ for 60 or 120 min resulted in the gradual accumulation of Bax into mitochondria, an effect completely inhibited by SP600125. In contrast, the abundance of both the cytosolic marker $\alpha$-tubulin and the mitochondrial marker citrate synthase in their respective cellular fraction was not altered by treatment with calphostin $\mathrm{C}$. Our results also indicated that Bax mitochondrial translocation correlated with a decrease in cell viability as suggested by trypan blue exclusion assay, and that exposure of cells to SP600125 completely abolished the ability of calphostin $\mathrm{C}$ to induce cell death (Figure 1c). Thus, these results are completely consistent with the idea that calphostin $\mathrm{C}$ promotes Bax translocation to mitochondria and death of NIH 3T3 cells through JNK activation.

Depletion of TG2 by shRNA antagonizes calphostin C-induced apoptosis. To investigate the role of TG2 in calphostin C-induced apoptosis, we first silenced the expression of endogenous TG2 by RNA interference in $\mathrm{NIH}$ 3T3 fibroblasts. Knockdown of TG2 was accomplished by infecting cells with a lentiviral vector carrying a short hairpin RNA (shRNA) that targets mouse and human TG2 mRNA (Sh-TG2). To exclude potential nonspecific effects, cells were also infected with an empty lentiviral vector or a lentiviral vector expressing a scrambled shRNA (Shscrambled). As depicted in Figure $2 a$, the Sh-TG2 construct abolished almost completely TG2 protein expression at $72 \mathrm{~h}$ after infection, whereas the empty or Sh-scrambled vector had no such effect. The intracellular level of actin was also unaffected by any of these constructs. Consistent with a reduction of TG2 expression, cells infected with Sh-TG2 showed significantly less basal transglutaminase activity than cells infected with the empty lentivirus (Figure 2b). The crosslinking activity remaining after TG2 knockdown was probably due to the presence, in these cells, of other transglutaminases, such as TG7, which are ubiquitously expressed ${ }^{11}$ or, alternatively, due to the residual levels of TG2 protein.

With the Sh-TG2-infected cells in hand, we next examined the effects of TG2 depletion on calphostin C-induced DLK oligomerization, activation of JNK and PARP cleavage by western blotting with specific antibodies. When compared with cells infected with the empty or Sh-scrambled lentivirus, in which we detected downregulation of DLK as early as $30 \mathrm{~min}$ after exposure to calphostin $\mathrm{C}$ and the appearance of DLK oligomers 45 min later, TG2-depleted NIH 3T3 fibroblasts 
a Cal (min) :
- JNK inhibitor

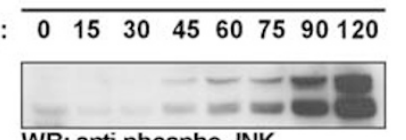

WB: anti-phospho-JNK
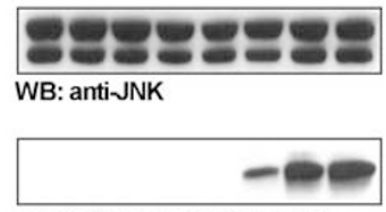

WB: anti-cleaved caspase-3

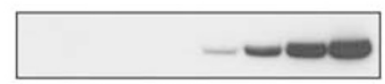

WB: anti-cleaved PARP

b

Cal (min) : $\frac{0 \quad 60120}{\text { Cytosol }} \frac{0 \quad 60120}{\text { Mitochondria }}$

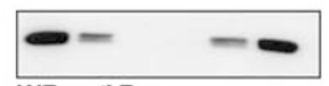

WB: anti-Bax

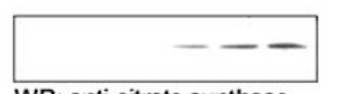

WB: anti-citrate synthase

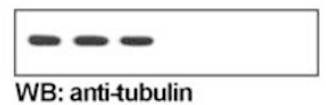

WB: anti-tubulin
+ JNK inhibitor

\begin{tabular}{llllllll}
\hline 0 & 15 & 30 & 45 & 60 & 75 & 90 & 120
\end{tabular}

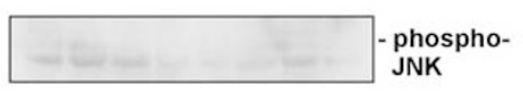

-
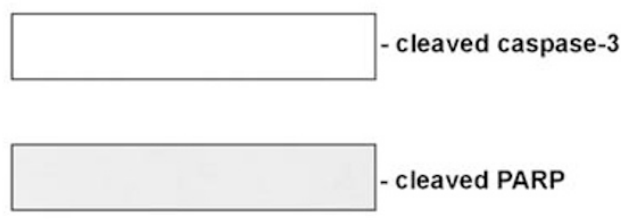

cleaved PARP

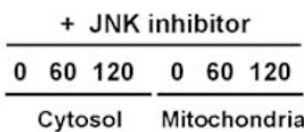
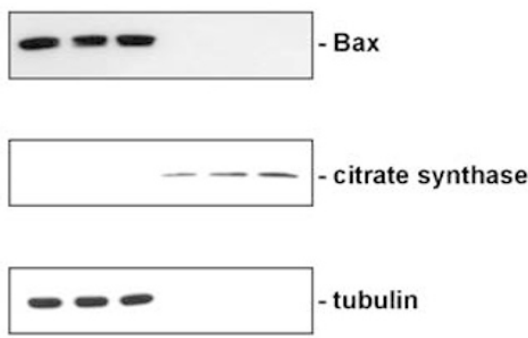

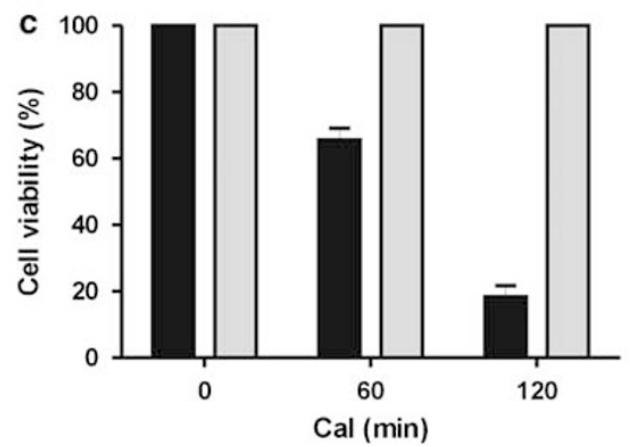

Figure 1 Calphostin C induces apoptosis in NIH 3 T3 cells through activation of the JNK signaling pathway. (a) NIH 3 T3 cells were incubated with or without the JNK inhibitor SP600125 $(20 \mu \mathrm{M})$ for $1 \mathrm{~h}$ prior to treatment with $250 \mathrm{nM}$ of calphostin C (Cal) for the time indicated. After incubation, cells were lysed and subjected to immunoblotting analysis with antibodies to JNK phosphorylated on Thr-183 and Tyr-185, JNK, cleaved caspase-3 and cleaved PARP. (b) NIH 3 T3 cells were incubated with or without the JNK inhibitor SP600125 $(20 \mu \mathrm{M})$ for $1 \mathrm{~h}$ prior to treatment with $250 \mathrm{nM}$ of calphostin C (Cal) for the time indicated. After incubation, cells were harvested and mitochondria were isolated using the Pierce mitochondria isolation kit. Cytosolic and mitochondrial fractions were run on SDS-PAGE and immunoblotted with antibodies to the pro-apoptotic protein Bax, the mitochondrial protein citrate synthase and the cytosolic protein tubulin. (c) NIH 3 T3 cells were incubated with or without the JNK inhibitor SP600125 (20 $\mu \mathrm{M}$ ) for $1 \mathrm{~h}$ prior to treatment with $250 \mathrm{nM}$ of calphostin $\mathrm{C}$ (Cal) for the time indicated. After incubation, cell viability was assessed by trypan blue dye exclusion assay. Values are means \pm S.D. $(n=3)$

showed impaired DLK oligomerization in response to calphostin $\mathrm{C}$ treatment (Figure 2c). Furthermore, knockdown of TG2 substantially diminished calphostin C-stimulated phosphorylation of JNK and cleavage of PARP, thereby supporting the hypothesis that TG2 mediates induction of DLK oligomerization, JNK activation and cell death by calphostin C. In agreement with this, ectopic expression of His-tagged wildtype active rat DLK in TG2-depleted NIH 3T3 cells neither induced nor promoted JNK activation and PARP cleavage in response to calphostin $C$ treatment (Figure $2 d$ ).

DLK knockdown prevents the induction of JNK phosphorylation and PARP cleavage by calphostin C. Since DLK is the only known protein kinase that serves as a substrate for TG2 in cells undergoing calphostin C-induced apoptosis, ${ }^{11}$ we tested the hypothesis that its 
a

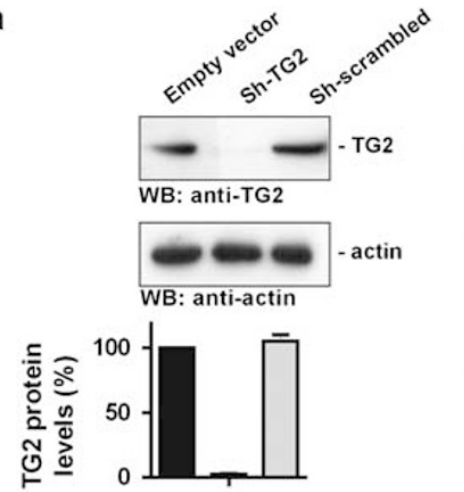

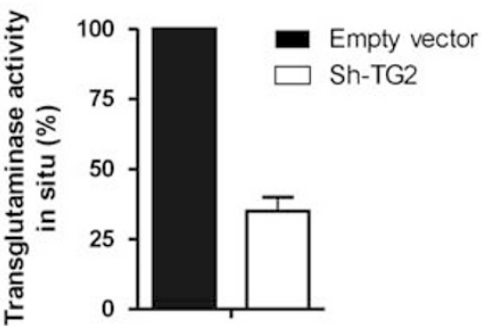

c Cal (min) : $\begin{array}{lllllll}0 & 15 & 30 & 456075 \quad 90120\end{array}$

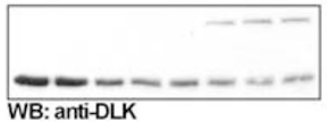
WB:anti-DIK

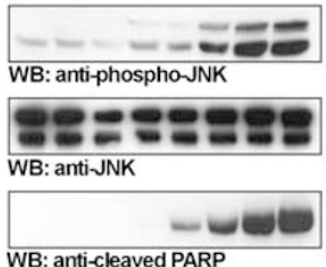
d
Cal $(\min ):$

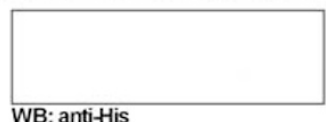

WB: anti-His

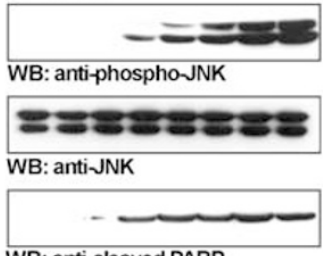

WB: anti-cleaved PARP
Sh-TG2
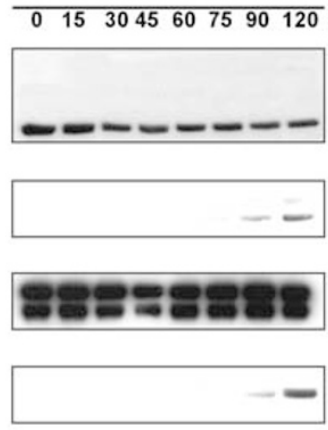

$\mathrm{Sh}-\mathrm{TG} 2+$ Empty vector

\begin{tabular}{lllllll}
\hline 0 & 15 & 3045 & 60 & 75 & 90 & 120
\end{tabular}
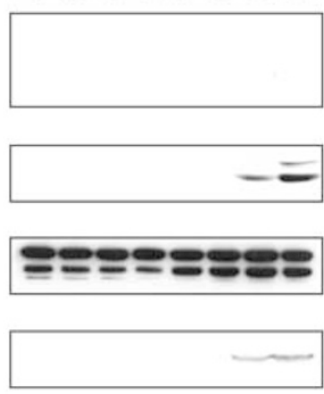

Sh-scrambled
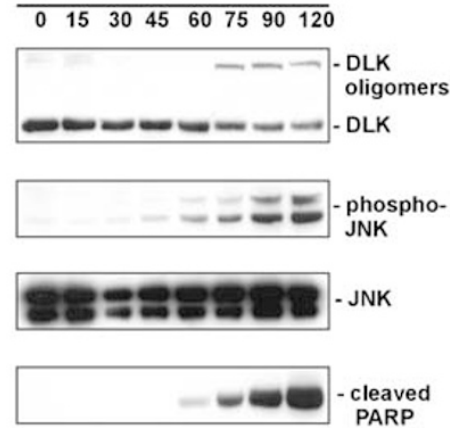

Sh-TG2 +

Sh-TG2
His-rDLK

\begin{tabular}{llllllll}
\hline 0 & 15 & 30 & 45 & 60 & 75 & 90 & 120
\end{tabular}
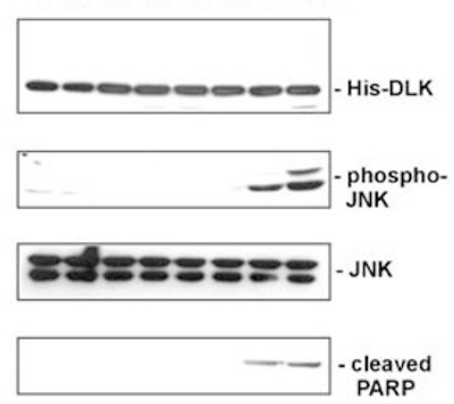

Figure 2 Knockdown of TG2 prevents calphostin C-induced DLK oligomerization, activation of JNK and PARP cleavage in NIH 3 T3 cells. (a) NIH 3 T3 cells were infected with an empty lentiviral vector or with a lentivirus expressing a TG2 shRNA or scrambled shRNA. Four days after infection, cells were lysed and subjected to immunoblotting analysis with an antibody directed against TG2. As a control for protein loading, immunoblots were probed in parallel with an antibody specific for $\gamma$-actin. TG2 protein levels were normalized to $\gamma$-actin levels. (b) NIH 3T3 cells infected with an empty lentiviral vector or a lentivirus expressing a TG2 shRNA were incubated with 5(biotinamido)pentylamine, and the incorporation of this polyamine derivative into proteins was used as a measure of in situ transglutaminase activity. Spectrophotometric analysis of 5-(biotinamido)pentylamine incorporation into substrate proteins was used to determine the level of transglutaminase activity. The data are expressed as a percent of transglutaminase activity relative to cells infected with the empty vector and are means \pm S.D. of three independent experiments. (c) NIH 3 T3 cells infected with an empty lentiviral vector or with a lentivirus expressing a TG2 shRNA or scrambled shRNA were incubated with $250 \mathrm{nM}$ calphostin C (Cal) for the indicated time periods, harvested and analyzed by immunoblotting with antibodies to DLK, phosphorylated JNK, JNK and cleaved PARP. (d) NIH 3T3 cells infected with an empty lentiviral vector or with a lentivirus expressing a TG2 shRNA were transiently transfected with empty vector $(15 \mu \mathrm{g})$ or an expression vector for His-tagged wild-type rat DLK (15 $\mu \mathrm{g})$. At $36 \mathrm{~h}$ after transfection, cells were exposed to $250 \mathrm{nM}$ calphostin C (Cal) for the indicated time periods, harvested and analyzed by immunoblotting with anti-His, anti-phospho-JNK, anti-JNK or anticleaved PARP antibodies

depletion by RNA interference would perturb the induction of JNK phosphorylation and PARP cleavage by calphostin C. Results shown in Figure $3 a$ demonstrate that lentivirusmediated delivery of mouse DLK shRNA (Sh-mDLK) attenuated by $\sim 80 \%$ the expression of DLK protein in NIH 3T3 cells. In contrast, infection with either an empty lentivirus or a lentivirus expressing a human DLK shRNA (Sh-hDLK no. 1), with four nucleotide mismatches compared to mouse DLK, was without effect. We next examined the consequences of DLK knockdown on calphostin C-induced JNK activation and PARP cleavage in empty vector, Sh-hDLK- or Sh-mDLK-infected cells that were exposed to calphostin $\mathrm{C}$ for various times. Western analysis with the phospho-JNK antibody revealed that NIH 3T3 cells with 


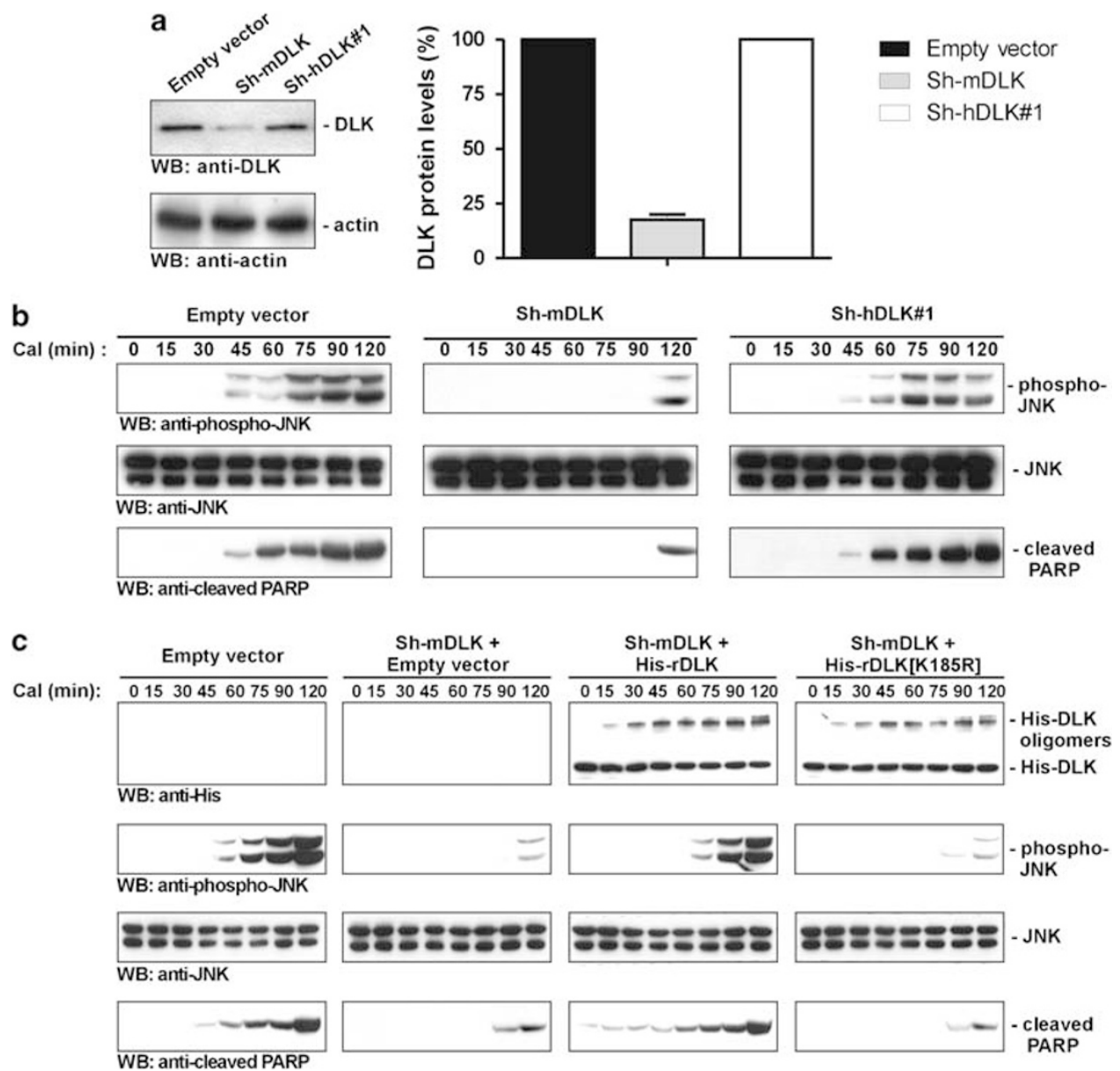

Figure 3 Depletion of DLK delays calphostin C-stimulated activation of JNK and PARP cleavage in NIH 3 T3 cells. (a) NIH 3 T3 cells were infected with an empty lentiviral vector or with a lentivirus expressing mouse or human DLK shRNA. Four days after infection, cells were lysed and subjected to immunoblotting analysis with an anti-DLK antibody. As a control for protein loading, immunoblots were probed in parallel with an antibody targeting $\gamma$-actin. DLK protein levels were normalized to $\gamma$-actin levels. (b) NIH 3 T3 cells infected with an empty lentiviral vector or with a lentivirus expressing mouse or human DLK shRNA were incubated with $250 \mathrm{nM}$ calphostin C (Cal) for the indicated time periods, harvested and analyzed by immunoblotting with antibodies to phosphorylated JNK, JNK and cleaved PARP. (c) NIH 3 T3 cells infected with an empty lentiviral vector or with a lentivirus expressing mouse DLK shRNA were transiently transfected with empty vector $(15 \mu \mathrm{g})$ or expression vectors for either His-tagged wild-type rat DLK $(15 \mu \mathrm{g})$ or His-tagged kinase-defective (K185R) rat DLK $(15 \mu \mathrm{g})$. At $24 \mathrm{~h}$ after transfection, cells were exposed to $250 \mathrm{nM}$ calphostin C (Cal) for the indicated time periods, harvested and analyzed by immunoblotting with anti-His, anti-phospho-JNK, anti-JNK or anti-cleaved PARP antibodies

lowered DLK protein levels were less sensitive than cells infected with either the empty or the Sh-hDLK lentivirus to the effects of calphostin C on JNK activity (Figure 3b). Indeed, calphostin $\mathrm{C}$ was able to stimulate the activation of $\mathrm{JNK}$ at $120 \mathrm{~min}$ in Sh-mDLK-infected cells compared to $45 \mathrm{~min}$ for the control cells. A similar delay in the response of Sh-mDLKinfected cells to calphostin $C$ was observed when western blotting was carried out with the antibody raised against cleaved PARP (Figure $3 b$ ). Therefore, these results suggest that DLK plays a major role in the cascade of events that links TG2 activation by calphostin C to stimulation of JNK activity and induction of cell death.

To further confirm a role for DLK in calphostin C-induced apoptosis, we performed a 'rescue' experiment in Sh-mDLKinfected cells that had been transiently transfected with an expression vector encoding His-tagged wild-type rat DLK, a recombinant protein resistant to silencing by the Sh-mDLK lentiviral vector employed here but sensitive to TG2-mediated oligomerization. Twenty-four hours after transfection, cells were treated with calphostin $C$ for various times, lysed and subjected to immunoblot analyses with antibodies against the His tag, phospho-JNK and cleaved PARP. As shown from our previous findings, depletion of DLK in Sh-mDLK-infected NIH 3T3 cells, which were transfected with an empty vector, resulted in activation of JNK and PARP cleavage at 90 or 120 min after exposure to calphostin $C$ compared to 45 or 60 min for empty vector-infected cells (Figure $3 c$ ). Importantly, reintroduction of an His-tagged wild-type rat DLK in these cells led to a small increase in the basal level of JNK activity and PARP cleavage, and this was further enhanced by calphostin C from 60 min onwards. Interestingly, despite its ability to oligomerize in response to calphostin $C$ like the wild-type rat DLK (Figure 3c), a catalytically inactive His-tagged rat DLK mutant failed to reverse the effects of DLK silencing on calphostin C-induced JNK activation and PARP cleavage. It thus appears that DLK, in its oligomerized and active form, acts as key regulator of the apoptotic response induced by calphostin C. 
Combined depletion of TG2 and DLK strongly inhibits calphostin C-induced JNK activation and cell death. Having observed that individual knockdown of TG2 or DLK significantly reduced JNK activation and PARP cleavage induction by calphostin $\mathrm{C}$, we then tested the effects of depleting both proteins in NIH 3T3 cells. As shown in Figure $4 \mathrm{a}$, cells infected with Sh-TG2 and Sh-mDLK lentiviruses showed a marked reduction of TG2 and DLK protein expression compared with the control. This combined depletion of TG2 and DLK proteins resulted in the complete loss of calphostin C-induced JNK activation within the time period examined together with inhibition of caspase-3 and PARP cleavage (Figure 4b). Consistent with these findings, shRNA knockdown of both TG2 and DLK completely abolished Bax translocation from cytosol to mitochondria in response to calphostin $C$ (Figure $4 \mathrm{c}$ ) and prevented the loss of cell viability more efficiently than knockdown of TG2 or DLK alone (Figure 4d). Together, these results show that TG2 and DLK are necessary for calphostin C action on JNK activity and cell death.
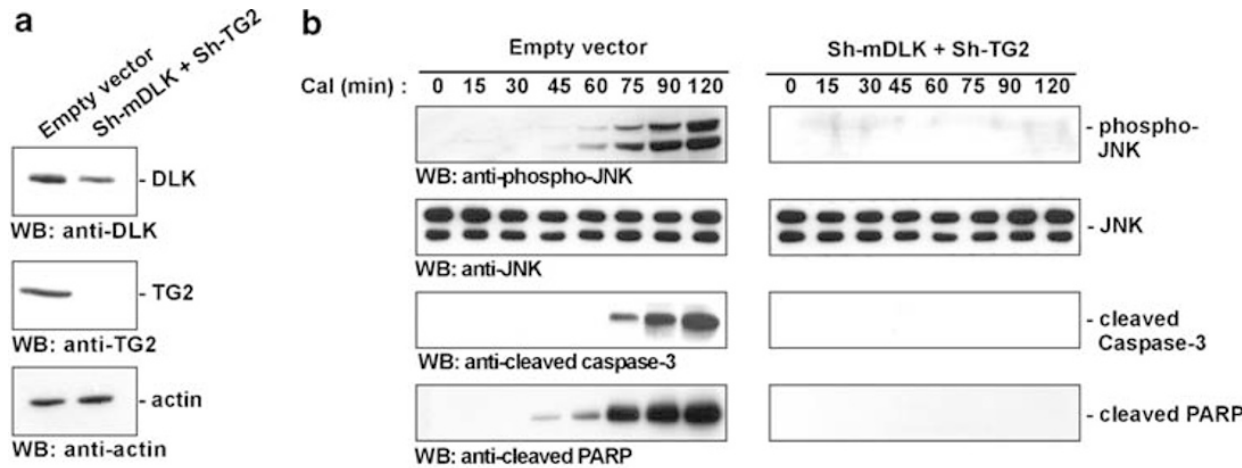

Cal $(\min )$ :
:
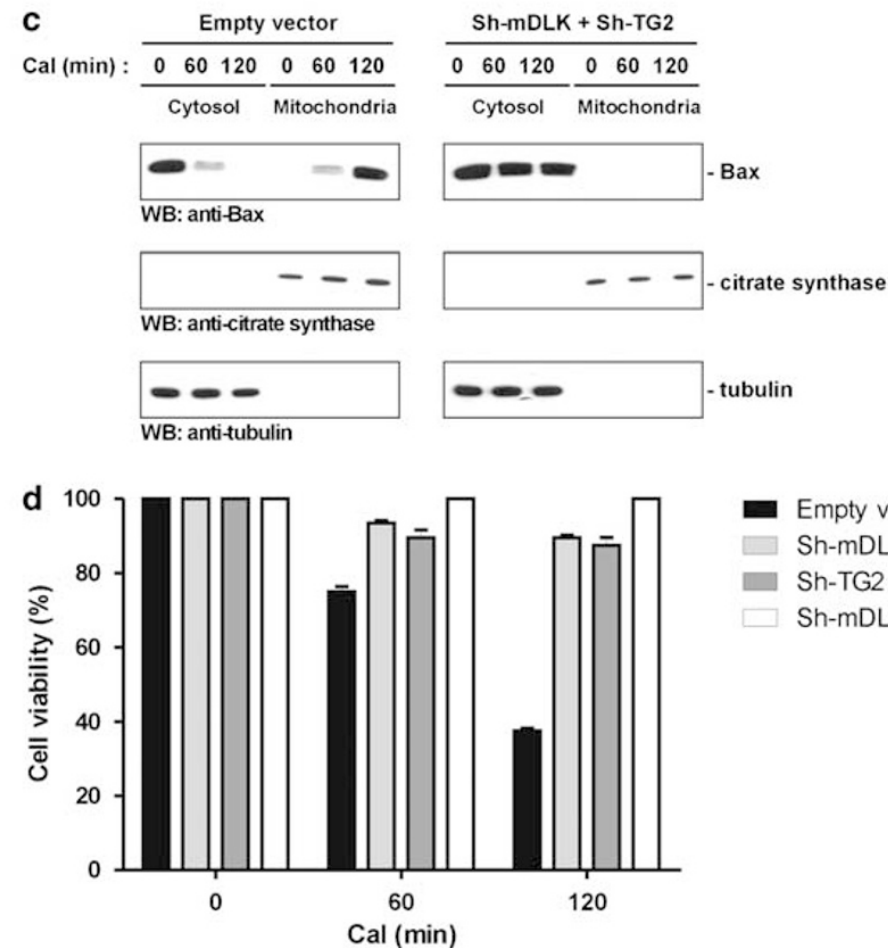

- tubulin

Empty vector

Sh-mDLK

Sh-TG2

Sh-mDLK + Sh-TG2

Figure 4 Combined knockdown of TG2 and DLK inhibits calphostin C-induced activation of JNK and cell death. (a) NIH 3 T3 cells were infected with an empty lentiviral vector or with a combination of lentiviruses expressing TG2 and mouse DLK shRNAs. Four days after infection, cells were harvested and analyzed by immunoblotting with antiDLK and anti-TG2 antibodies. As a control for protein loading, immunoblots were probed in parallel with an antibody targeting $\gamma$-actin. (b) NIH 3 T3 cells were infected with an empty lentiviral vector or with a combination of lentiviruses expressing TG2 and mouse DLK shRNAs. Four days after infection, cells were incubated with $250 \mathrm{nM}$ calphostin C (Cal) for the time indicated, harvested and analyzed by immunoblotting with antibodies to phosphorylated JNK, JNK, cleaved caspase-3 and cleaved PARP. (c) NIH 3T3 cells infected with an empty lentiviral vector or with a combination of lentiviruses expressing TG2 and mouse DLK shRNAs were incubated with $250 \mathrm{nM}$ calphostin C (Cal) for the indicated time periods, harvested and mitochondria were isolated using the Pierce mitochondria isolation kit. Cytosolic and mitochondrial fractions were run on SDS-PAGE and immunoblotted with antibodies against the pro-apoptotic protein Bax, the mitochondrial protein citrate synthase and the cytosolic protein tubulin. (d) NIH 3T3 cells infected with an empty lentiviral vector, a lentivirus expressing TG2 shRNA, a lentivirus expressing mouse DLK shRNA or a combination of lentiviruses expressing TG2 and mouse DLK shRNAs were incubated with $250 \mathrm{nM}$ calphostin C (Cal) for the time indicated. After incubation, cell viability was assessed by trypan blue dye exclusion assay. Values are means \pm S.D. $(n=3)$ 
Calphostin C-induced apoptosis of breast cancer cells is mediated by the TG2-DLK-JNK pathway. To further explore the biological relevance of the preceding results, we next investigated the contribution of TG2, DLK and JNK to calphostin C-induced apoptosis using as a model system the drug-resistant and metastatic human breast cancer cell line MDA-MB-231, which expresses high basal levels of TG2. ${ }^{29}$ This issue was first addressed by examining in these cells whether calphostin $C$ induces JNK activation and PARP cleavage, as it does in mouse NIH 3 T3 fibroblasts, and whether pharmacological inhibition of JNK with SP600125 reverses its effects. Results shown in Figure 5 a indicate that calphostin $\mathrm{C}$ was able to stimulate phosphorylation of JNK and PARP cleavage as early as $1 \mathrm{~h}$ after treatment of MDA-MB-231 cells. However, prior incubation with SP600125 substantially reduced calphostin C effects on JNK phosphorylation and PARP cleavage, indicating that JNK activation is sufficient to trigger the induction of apoptosis by this compound.

MDA-MB-231 cells infected with control lentivirus or lentivirus expressing shRNA against either TG2 (Sh-TG2) or human DLK (Sh-hDLK no. 2) were then used to examine how a

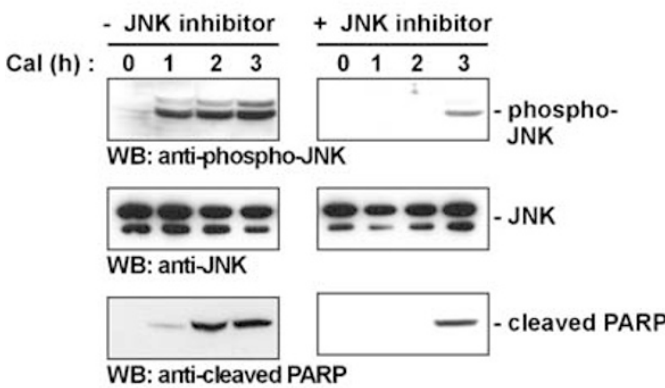

b
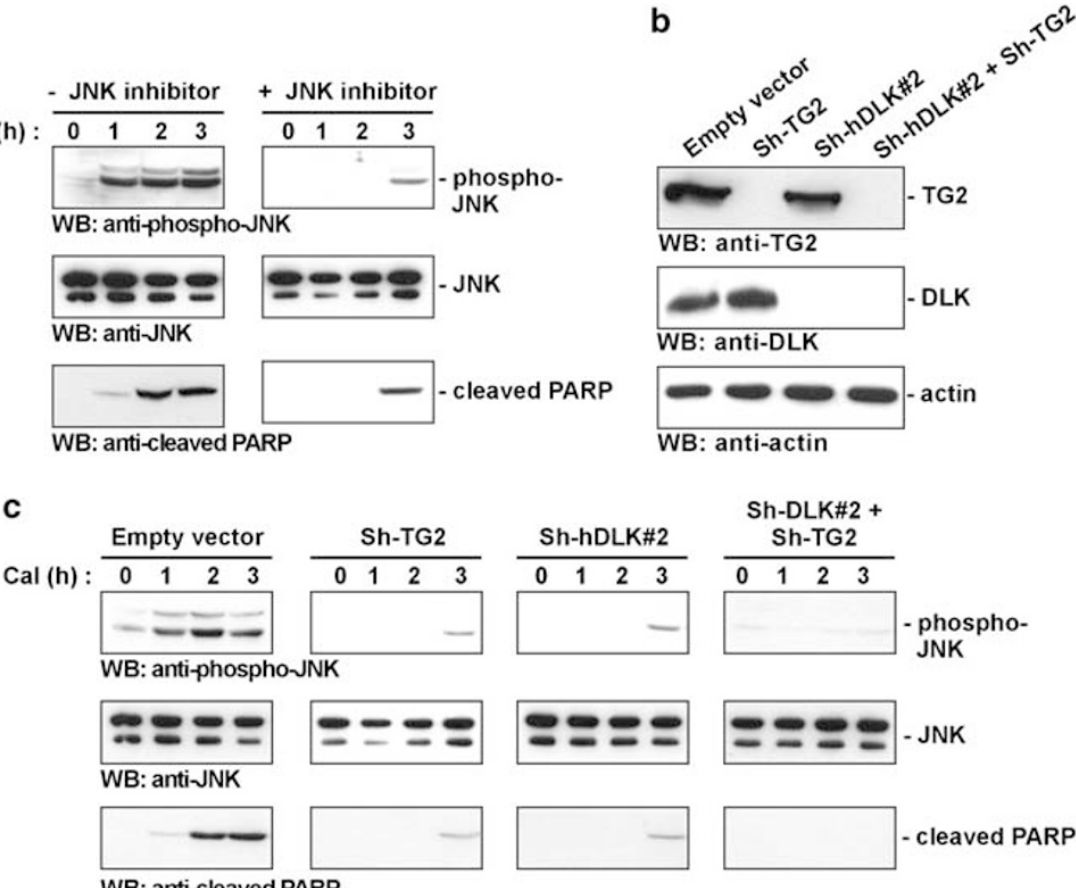

WB: anti-cleaved PARP
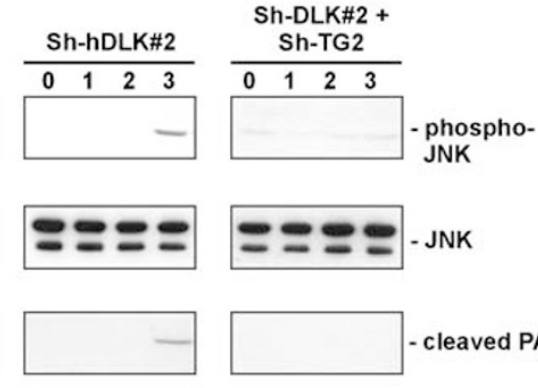

cleaved PARP

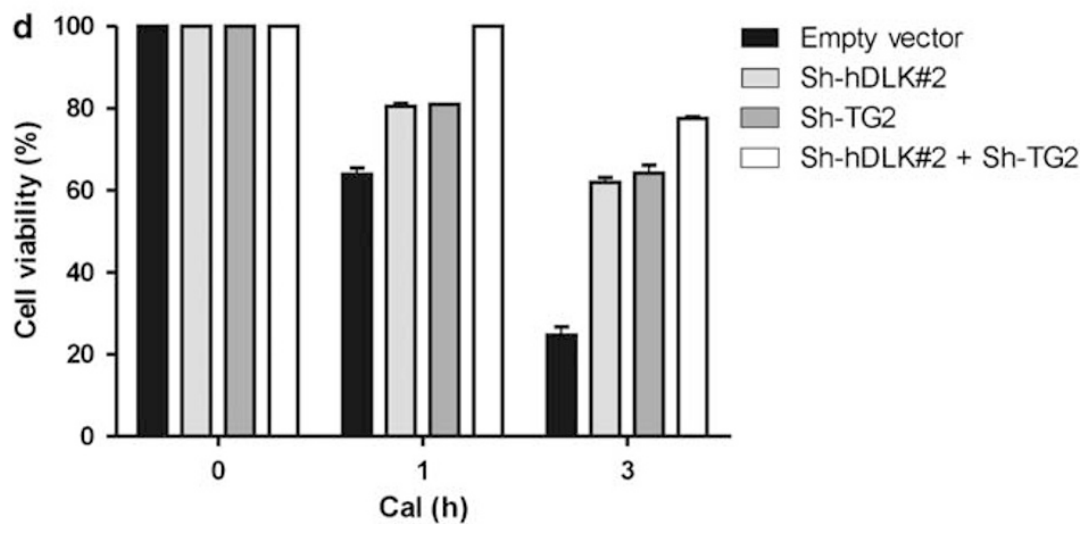

Figure 5 Calphostin C-induced apoptosis of breast cancer cells is mediated by TG2, DLK and JNK. (a) MDA-MB-231 breast cancer cells were incubated with or without the JNK inhibitor SP600125 $(20 \mu \mathrm{M})$ for $2 \mathrm{~h}$ prior to treatment with $250 \mathrm{nM}$ of calphostin $\mathrm{C}$ (Cal) for the time indicated. After incubation, cells were lysed and subjected to immunoblotting analysis with antibodies to phosphorylated JNK, JNK and cleaved PARP. (b) MDA-MB-231 cells were infected with an empty lentiviral vector, a lentivirus expressing TG2 shRNA, a lentivirus expressing human DLK shRNA or a combination of lentiviruses expressing TG2 and human DLK shRNAs. Four days after infection, cells were harvested and analyzed by immunoblotting with anti-DLK and anti-TG2 antibodies. As a control for protein loading, immunoblots were probed in parallel with an antibody targeting $\gamma$-actin. (c) MDA-MB-231 cells infected with either an empty lentiviral vector, a lentivirus expressing TG2 shRNA, a lentivirus expressing human DLK shRNA or a combination of lentiviruses expressing TG2 and human DLK shRNAs were incubated with $250 \mathrm{nM}$ calphostin C (Cal) for the indicated time periods, harvested and analyzed by immunoblotting with antibodies to phosphorylated JNK, JNK and cleaved PARP. (d) MDA-MB-231 cells infected with either an empty lentiviral vector, a lentivirus expressing TG2 shRNA, a lentivirus expressing human DLK shRNA or a combination of lentiviruses expressing TG2 and human DLK shRNAs were incubated with $250 \mathrm{nM}$ calphostin C (Cal) for the time indicated. After incubation, cell viability was assessed by trypan blue dye exclusion assay. Values are means \pm S.D. $(n=3)$ 
the absence of TG2, DLK or both proteins affects calphostin $\mathrm{C}$-induced apoptosis. As shown in Figure 5b, the constructs efficiently and specifically knocked down expression of the endogenous TG2 and DLK in this model. Immunoblot analysis demonstrated that the knockdown of either TG2 or DLK strongly reduced calphostin $C$ effects on JNK activity and PARP within the time period examined, although the combined depletion of these two enzymes further altered this response (Figure 5c). As expected from our studies in $\mathrm{NIH}$ 3T3 fibroblasts, the combined knockdown of TG2 and DLK in MDA-MB-231 cells also conferred better survival in response to calphostin $C$ treatment than did depletion of TG2 or DLK alone (Figure $5 d$ ). Taken together, these results indicate that calphostin C needs both TG2 and DLK to fully transduce its apoptotic signal in MDA-MB-231 cells.

\section{Discussion}

Calphostin C-mediated cell death has been previously reported to be associated with the TG2-dependent oligomerization and activation of DLK, an upstream component of the JNK signaling pathway. ${ }^{9}$ In the present study, we used mouse NIH 3T3 fibroblasts and human MDA-MB-231 breast cancer epithelial cells as in vitro model systems to investigate the role of TG2, DLK and JNK in the mechanisms underlying cell death induced by calphostin $C$. Our data indicate that calphostin $C$ exposure causes JNK activation, concomitantly with induction of apoptosis, as evidenced by Bax translocation to mitochondria, cleavage of caspase-3 and PARP as well as loss of cell viability. Interestingly, JNK seems to be indispensable for calphostin $\mathrm{C}$ action on apoptosis as inhibition of its activity by SP600125 markedly reduced this response. Thus, these results strongly suggest that $\mathrm{JNK}$, in its active form, acts as an important mediator of calphostin C-induced apoptosis in NIH 3T3 fibroblasts and MDA-MB-231 epithelial cells. The notion that JNK might contribute to the pro-apoptotic properties of calphostin $C$ is not completely unexpected because prior studies have demonstrated the existence of a tight connection between activation of this protein and apoptosis induction in cells exposed to a wide array of stresses, including UV irradiation, heat shock, proinflammatory cytokines and chemotherapeutic drugs. ${ }^{18,30}$ The involvement of JNK in apoptosis is also supported by the finding that primary fibroblasts prepared from JNK-deficient mouse embryos are resistant to cell death induced by UV irradiation. ${ }^{31}$

Using an RNA-interference strategy, we found that the ability of calphostin $\mathrm{C}$ to induce JNK activation and apoptosis in NIH 3T3 fibroblasts and MDA-MB-231 cells was considerably reduced after TG2 depletion. This observation indicates that TG2 is a key component of the signaling pathway leading to JNK activation and induction of apoptotic cell death by calphostin C. Previous studies have established that TG2 modulates apoptosis in a stimulus-dependent manner. Data from Tucholski and Johnson ${ }^{13}$ suggest that TG2 is proapoptotic when cells are exposed to stressors that increase its $\mathrm{Ca}^{2+}$-dependent transamidating activity, but antiapoptotic under conditions in which its crosslinking activity remains latent. Several apoptotic stimuli, such as osmotic stress, ${ }^{13}$ oxidative stress ${ }^{32}$ and UV irradiation, ${ }^{32}$ have been shown to activate the in situ crosslinking function of TG2, presumably due to an increase in intracellular concentrations of calcium. As calphostin $\mathrm{C}$ is another stressor able to activate the crosslinking function of $\mathrm{TG}_{2},^{9}$ the mechanism of TG2mediated activation of JNK and apoptosis is likely due to the polymerization of various protein substrates involved in the apoptotic process. Of all the TG2 substrates identified so far, ${ }^{11}$ DLK is the only one with an established role in JNK activation, ${ }^{33,34}$ and its polymerization has been found to facilitate cell commitment to apoptosis in response to calphostin $\mathrm{C}^{9}{ }^{9}$ This suggests that DLK might act as an effector of TG2 during calphostin C-induced apoptosis. Support for such a role for DLK is provided by our observations that (1) TG2 knockdown prevents the accumulation of DLK oligomers in response to calphostin C; (2) depletion of DLK markedly reduces calphostin $\mathrm{C}$-induced JNK activation and PARP cleavage; and (3) reintroduction of wild-type rat DLK, but not kinase-defective DLK, in mouse-silenced NIH 3T3 cells restores calphostin $\mathrm{C}$ ability to stimulate JNK activity and PARP cleavage. Collectively, these results favor a model in which calphostin $C$ induces apoptosis through a sequential pathway involving TG2, DLK, JNK and mitochondria (Figure 6). Such a scenario is consistent with the previous observations that activation of TG2 by calphostin $C$ leads to the oligomerization and stimulation of DLK, which, in turn, promotes the activation of JNK. ${ }^{9}$ Further support for the idea that TG2 and DLK function in a linear pathway also derives from the present finding that combined knockdown of both proteins has additive effects in lowering JNK activation and cell death by calphostin C, compared to TG2 or DLK knockdown alone.

In conclusion, our current findings provide the first genetic evidence that TG2 and DLK play an essential role in the execution of calphostin C-induced apoptosis. Although the participation of other signaling pathways and kinases in this process cannot be excluded at present, our results also underscore the absolute requirement of JNK activity in regulation of TG2-mediated calphostin C-induced apoptosis. Understanding the mechanisms of apoptosis caused by TG2 has fundamental biological interest, because expression of TG2 and activation of its crosslinking function have been linked to both the development of neurodegenerative diseases $^{35-37}$ and the induction of death in drug-resistant cancer cells. ${ }^{38,39}$ Studies directed at identifying whether DLK forms homodimers and/or heterodimers with another cytosolic substrate in calphostin-treated cells should also provide valuable insights into the mechanisms by which DLK transduces an apoptotic signal.

\section{Materials and Methods}

Chemicals and antibodies. Calphostin C and SP600125 were purchased from Calbiochem-Novabiochem Co. (San Diego, CA, USA). Protease inhibitors and all other common reagents were obtained from Sigma-Aldrich Canada Ltd. (Oakville, ON, USA). BAP (5-(biotinamido)pentylamine) and horseradish peroxidase (HRP)-conjugated streptavidin were from Pierce Biotechnology (Rockford, IL, USA). The rabbit polyclonal antiserum to DLK was described previously. ${ }^{40}$ Rabbit polyclonal antibodies raised against cleaved caspase-3 and cleaved PARP were purchased from Cell Signaling Technology Inc. (Beverly, MA, USA). The rabbit polyclonal and the mouse monoclonal antibodies against $\gamma$-actin and $\alpha$-tubulin were obtained from Sigma-Aldrich Ltd. The rabbit polyclonal antibodies against Bax and TG2 as well as the mouse monoclonal antibodies against phospho-JNK and total JNK were from Santa Cruz Biotechnology Inc. (Santa Cruz, CA, USA.). Finally, the 


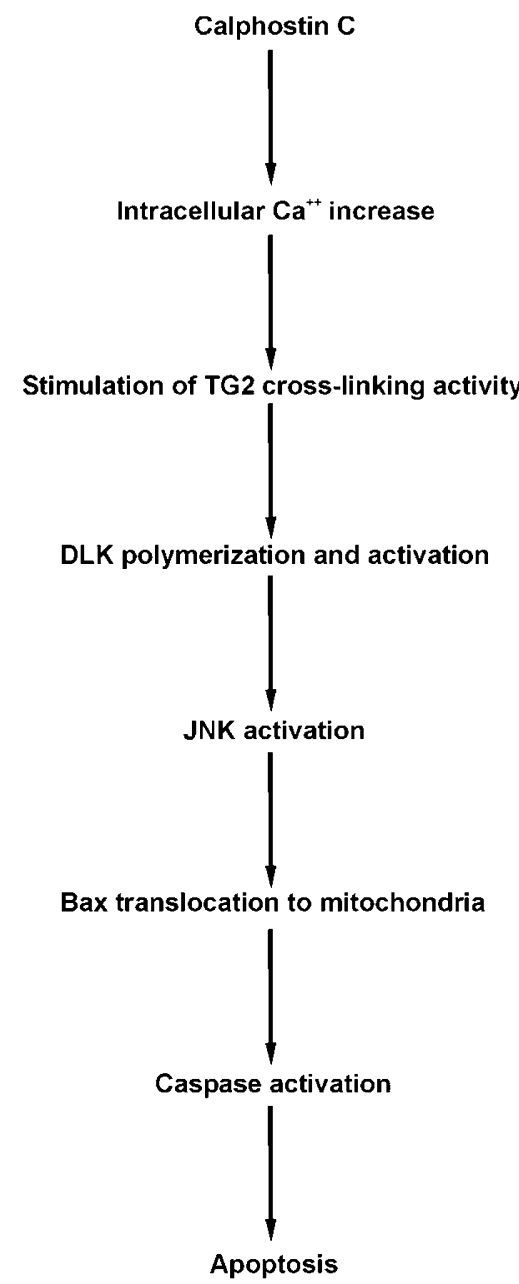

Figure 6 Proposed model for the regulation of calphostin C-induced apoptosis by TG2, DLK and JNK. In NIH 3T3 and MDA-MB-231 cells, calphostin C activates the crosslinking function of TG2 as a result of its ability to induce calcium mobilization. Once activated, TG2 catalyzes the formation of DLK oligomers capable of causing sustained activation of JNK, which in turn promotes Bax translocation to mitochondria and the subsequent caspase-dependent apoptosis. It is worth noting that the possibility that calphostin C triggers a TG2-independent pathway to induce cell death beyond the time period examined cannot be ruled out completely

citrate synthase antibody was generously provided by Mr. Gilles Grondin (Université de Sherbooke, Qc, Canada). Cell culture reagents were from Invitrogen Corp. (Carlsbad, CA, USA), HyClone Laboratories (Logan, UT, USA) and Wisent Inc. (St-Bruno, Qc, Canada).

Cell culture. Mouse NIH 3T3 fibroblasts were cultured in Dulbecco's modified Eagle's medium (DMEM) supplemented with $5 \%$ (v/v) cosmic calf serum (CCS), $100 \mathrm{U} / \mathrm{ml}$ penicillin, $100 \mu \mathrm{g} / \mathrm{ml}$ streptomycin and $25 \mu \mathrm{g} / \mathrm{ml}$ amphotericin B. Human MDA-MB-231 breast cancer cells were maintained in DMEM supplemented with $10 \%$ (v/v) fetal bovine serum (CCS), $100 \mathrm{U} / \mathrm{ml}$ penicillin, $100 \mu \mathrm{g} / \mathrm{ml}$ streptomycin and $25 \mu \mathrm{g} / \mathrm{ml}$ amphotericin B. Both cell lines were maintained at $37^{\circ} \mathrm{C}$ in a humidified atmosphere consisting of $5 \% \mathrm{CO}_{2} / 95 \%$ air. Exposure to calphostin $\mathrm{C}(250 \mathrm{nM})$ was routinely carried out on late log (i.e. $90 \%$ confluent) cultures, in the continuous presence of fluorescent light. In some experiments (see Results), cells were preincubated with the JNK inhibitor SP600125 $(20 \mu \mathrm{M})$ for 1 or $2 \mathrm{~h}$ before the addition of calphostin $\mathrm{C}$. The final concentrations of diluent/vehicle (dimethylsulfoxide) never exceeded $0.1 \%(\mathrm{v} / \mathrm{v})$.
Preparation of cell lysates and immunoblotting. Cells were lysed for $60 \mathrm{~min}$ at $4{ }^{\circ} \mathrm{C}$ in $15 \mathrm{mM}$ Tris- $\mathrm{HCl} \mathrm{pH} \mathrm{7.4,1 \%}$ Triton X-100, $0.2 \%$ sodium dodecyl sulfate (SDS), $0.5 \%$ sodium deoxycholate, $150 \mathrm{mM} \mathrm{NaCl}, 1 \mathrm{mM} \mathrm{MgCl}$, $1 \mathrm{mM}$ EGTA, $10 \mathrm{mM} \beta$-mercaptoethanol, $0.2 \mathrm{mM}$ sodium orthovanadate, $1 \mathrm{mM}$ phenylmethylsulfonyl fluoride (PMSF), $1 \mu \mathrm{g} / \mathrm{ml}$ leupeptin and $1 \mu \mathrm{g} / \mathrm{ml}$ aprotinin. Lysates were clarified by centrifugation $\left(12000 \times g\right.$ for $10 \mathrm{~min}$ at $\left.4^{\circ} \mathrm{C}\right)$, and the concentration of total protein in the supernatant fraction was quantified by the modified Bradford protein assay (Bio-Rad Laboratories, Mississauga, Ontario, Canada). For immunoblotting, equal amounts of proteins were fractionated by SDS-polyacrylamide gel electrophoresis (SDS-PAGE) and transferred onto polyvinylidene difluoride (PVDF) membranes (Roche Diagnostics, Laval, Québec, Canada) using a semidry transfer apparatus (Bio-Rad Laboratories). Membranes were blocked for $1 \mathrm{~h}$ at room temperature in $20 \mathrm{mM}$ Tris, $\mathrm{pH} 7.5,150 \mathrm{mM} \mathrm{NaCl}$, $0.1 \%$ Tween 20 containing $5 \%$ skim milk powder before overnight incubation with the primary antibody at $4{ }^{\circ} \mathrm{C}$. Immunoreactive bands were detected by enhanced chemiluminescence using secondary HRP-conjugated antibodies (ECL Plus Western blotting kit; Amersham Pharmacia Biotech Inc.).

Mitochondria isolation. Mitochondria were isolated from parental NIH 3T3 and Sh-TG2 + Sh-mDLK-infected NIH 3T3 cells incubated in the presence or absence of SP600125 and/or calphostin C using a mitochondria isolation kit from Pierce Biotechnology. The mitochondrial fractions were boiled in Laemli buffer, separated by SDS-PAGE and transferred to PVDF membranes for immunoblotting.

Cell viability assay. Control or infected NIH 3 T3 and MDA-MB-231 cells incubated in the absence or presence of calphostin C $(250 \mathrm{nM})$ were harvested with trypsin followed by staining with $0.4 \%$ trypan blue dye. The percentage of viable cells was determined using a hemocytometer. Cell viability (\%) means the ratio of the number of trypan blue-impermeable cells to the total cell counts (trypan blueimpermeable cell number/total cell number).

Construction of lentiviral vectors. Oligonucleotides encoding mouse and human TG2 (5'-GAGCGAGATGATCTGGAAC-3', Sh-TG2), mouse DLK (5'-AGCCTACTCCACAGAACAC-3', Sh-mDLK), human DLK (5'-ACCGACATCAA GCACTTGC- $3^{\prime}$, underlined bases are not conserved in mouse, Sh-hDLK no. 1) and scramble $\bar{d}$ (5'-TGCTGACTCCAAAGCTCTG-3', Sh-scrambled) short hairpin sequences were cloned into the lentivirus-based RNAi vector pLVTHM (Addgene Inc., Cambridge, MA, USA) according to the protocol of Dr. Didier Trono (University of Geneva Medical School, Geneva, Switzerland, http://tronolab.epfl.ch). A lentiviral vector encoding a human DLK (5'-CTACGACGATGTGGTGAAGATCTC-3') short hairpin sequence, designated herein Sh-hDLK no. 2, was also purchased from Open Biosystems (Huntsville, AL, USA) and used for experiments in the human breast cancer cell line MDA-MB-231.

Lentivirus infection. Lentiviral stocks were prepared as previously reported using 293T cells as packaging cells (see http://www.tronolab.epfl. ch for details). Subconfluent NIH 3T3 and MDA-MB-231 cells were incubated with viral supernatants for $24 \mathrm{~h}$, washed twice with phosphate-buffered saline (PBS) and incubated with normal medium for an additional 2 days. Then, the cells were treated with calphostin $\mathrm{C}$ for the indicated time points, harvested and processed for further analyses.

In situ TG2 activity assay. Empty vector- and Sh-TG2-infected NIH 3T3 cells were labeled for $2 \mathrm{~h}$ with $1 \mathrm{mM}$ BAP. The cells were subsequently rinsed twice with PBS, harvested in lysis buffer $(50 \mathrm{mM}$ Tris- $\mathrm{HCl}$ pH 7.5, $0.3 \mathrm{M} \mathrm{NaCl}, 5 \mathrm{mM}$ EGTA, $1 \mathrm{mM}$ EDTA, $0.5 \%$ Triton X-100, 0.5\% NP-40, $0.1 \mathrm{mM}$ PMSF, $1 \mu \mathrm{g} / \mathrm{ml}$ leupeptin and $1 \mu \mathrm{g} / \mathrm{ml}$ aprotinin) and sonicated on ice. Protein concentration in the lysates were determined by a modified Bradford protein assay (Bio-Rad Laboratories). TG2 activity was quantified by measuring the transglutaminase-dependent incorporation of BAP into proteins by a microplate assay as described previously. ${ }^{41}$

Plasmids and transfection. The full-length coding sequence of wild-type rat DLK, which shows one nucleotide mismatch with the mouse DLK shRNA described here, was inserted in frame with an His tag sequence in the PTriEx-4 expression vector (Novagen Inc., Madison, WI, USA). The expression vector for His-tagged catalytically inactive rat DLK was produced by replacing the invariant lysine at position 185 within kinase subdomain II with arginine using the Stratagene QuickChange site-directed mutagenesis kit (La Jolla, CA, USA). For the 'rescue' experiment, NIH 3 T3 cells were infected with an empty lentiviral vector or a lentiviral 
vector expressing mouse DLK shRNA and transfected, $48 \mathrm{~h}$ later, with the different expression vectors mentioned above using PolyFect transfection reagent (Qiagen, Valencia, CA, USA). Twenty-four hours after transfection, cells were treated with calphostin $\mathrm{C}(250 \mathrm{nM})$ for the indicated time points, harvested and processed for further analyses.

Acknowledgements. We thank Dr. Alain Lavigueur for critical reading of the article and Dr. Nicolas Gévry for his help with the design of the short hairpin sequences. This work was supported by a grant from the Canadian Institutes of Health Research (ClHR). KR was recipient of a studentship from the Fonds Québécois de la Recherche sur la Nature et les Technologies.

1. Kobayashi E, Nakano H, Morimoto M, Tamaoki T. Calphostin C (UNC-1028C), a novel microbial compound, is a highly potent and specific inhibitor of protein kinase C. Biochem Biophys Res Commun 1989; 159: 548-553.

2. Ikemoto H, Tani E, Matsumoto T, Nakano A, Furuyama J. Apoptosis of human glioma cells in response to calphostin C, a specific protein kinase C inhibitor. J Neurosurg 1995; 83: 1008-1016.

3. Zhu DM, Narla RK, Fang WH, Chia NC, Uckun FM. Calphostin C triggers calciumdependent apoptosis in human acute lymphoblastic leukemia cells. Clin Cancer Res 1998; 4: 2967-2976.

4. Ozaki I, Tani E, Ikemoto H, Kitagawa H, Fujikawa $\mathrm{H}$. Activation of stress-activated protein kinase/c-Jun NH2-terminal kinase and p38 kinase in calphostin C-induced apoptosis requires caspase-3-like proteases but is dispensable for cell death. J Biol Chem 1999; 274 5310-5317.

5. Ikemoto H, Tani E, Ozaki I, Kitagawa H, Arita N. Calphostin C-mediated translocation and integration of Bax into mitochondria induces cytochrome $\mathrm{c}$ release before mitochondrial dysfunction. Cell Death Differ 2000; 7: 511-520.

6. Zheng XL, Gui Y, Du G, Frohman MA, Peng DQ. Calphostin-C induction of vascular smooth muscle cell apoptosis proceeds through phospholipase $D$ and microtubule inhibition. J Biol Chem 2004; 279: 7112-7118.

7. Oliverio S, Amendola A, Di Sano F, Farrace MG, Fesus L, Nemes $Z$ et al. Tissue transglutaminase-dependent posttranslational modification of the retinoblastoma gene product in promonocytic cells undergoing apoptosis. Mol Cell Biol 1997; 17: 6040-6048.

8. Hébert SS, Daviau A, Grondin G, Latreille M, Aubin RA, Blouin R. The mixed lineage kinase DLK is oligomerized by tissue transglutaminase during apoptosis. I Biol Chem 2000; 275: 32482-32490.

9. Robitaille K, Daviau A, Tucholski J, Johnson GV, Rancourt C, Blouin R. Tissue transglutaminase triggers oligomerization and activation of dual leucine zipper-bearing kinase in calphostin C-treated cells to facilitate apoptosis. Cell Death Differ 2004; 11: $542-549$.

10. Lorand L, Graham RM. Transglutaminases: crosslinking enzymes with pleiotropic functions. Nat Rev Mol Cell Biol 2003; 4: 140-156.

11. Esposito C, Caputo I. Mammalian transglutaminases. Identification of substrates as a key to physiological function and physiopathological relevance. FEBS J 2005; 272: 615-631.

12. Fésüs $L$, Szondy $Z$. Transglutaminase 2 in the balance of cell death and survival. FEBS Lett 2005; 579: 3297-3302.

13. Tucholski J, Johnson GVW. Tissue transglutaminase differentially modulates apoptosis in a stimuli-dependent manner. J Neurochem 2002; 81: 780-791.

14. Antonyak MA, Singh US, Lee DA, Boehm JE, Combs C, Zgola MM et al. Effects of tissue transglutaminase on retinoic acid-induced cellular differentiation and protection against apoptosis. J Biol Chem 2001; 276: 33582-33587.

15. Antonyak MA, Boehm JE, Cerione RA. Phosphoinositide 3-kinase activity is required for retinoic acid-induced expression and activation of the tissue transglutaminase. J Biol Chem 2002; 277: 14712-14716.

16. Nemes Jr Z, Adány R, Balázs M, Boross P, Fésüs L. Identification of cytoplasmic actin as an abundant glutaminyl substrate for tissue transglutaminase in $\mathrm{HL}-60$ and U937 cells undergoing apoptosis. J Biol Chem 1997; 272: 20577-20583.

17. Ballestar E, Abad C, Franco L. Core histones are glutaminyl substrates for tissue transglutaminase. J Biol Chem 1996; 271: 18817-18824.

18. Davis RJ. Signal transduction by the JNK group of MAP kinases. Cell 2000; 103: 239-252.
19. Harper SJ, LoGrasso P. Signalling for survival and death in neurones-The role of stressactivated kinases, JNK and P38. Cell Signalling 2001; 13: 299-310.

20. Yang DD, Kuan CY, Whitmarsh AJ, Rincon M, Zheng TS, Davis RJ et al. Absence of excitotoxicity-induced apoptosis in the hippocampus of mice lacking the Jnk3 gene. Nature 1997; 389: 865-870.

21. Troy CM, Rabacchi SA, Xu Z, Maroney AC, Connors TJ, Shelanski ML et al. beta-Amyloidinduced neuronal apoptosis requires $\mathrm{C}$-Jun $\mathrm{N}$-terminal kinase activation. J Neurochem 2001; 77: 157-164.

22. Maroney AC, Finn JP, Bozyczko-Coyne D, O'Kane TM, Neff NT, Tolkovsky AM et al. CEP. 1347 (KT7515), an inhibitor of JNK activation, rescues sympathetic neurons and neuronally differentiated PC12 cells from death evoked by three distinct insults. J Neurochem 1999; 73: $1901-1912$.

23. Mayne GC, Murray AW. Evidence that protein kinase $\mathrm{C}$ epsilon mediates phorbol ester inhibition of calphostin C- and tumor necrosis factor-alpha-induced apoptosis in U937 histiocytic lymphoma cells. J Biol Chem 1998; 273: 24115-24121.

24. Pollack IF, Kawecki S. The effect of calphostin $\mathrm{C}$, a potent photodependent protein kinase C inhibitor, on the proliferation of glioma cells in vitro. J Neurooncol 1997; 31: 255-266.

25. Porter AG, Jänicke RU. Emerging roles of caspase-3 in apoptosis. Cell Death Differ 1999; 6: 99-104.

26. Lazebnik YA, Kaufmann SH, Desnoyers S, Poirier GG, Earnshaw WC. Cleavage of poly(ADP-ribose) polymerase by a proteinase with properties like ICE. Nature 1994; 371: 346-347.

27. Bennett BL, Sasaki DT, Murray BW, O'Leary EC, Sakata ST, Xu W et al. SP600125, an anthrapyrazolone inhibitor of Jun N-terminal kinase. Proc Natl Acad Sci USA 2001; 98: 13681-13686

28. Kim BJ, Ryu SW, Song BJ. JNK- and p38 kinase-mediated phosphorylation of Bax leads to its activation and mitochondrial translocation and to apoptosis of human hepatoma HepG2 cells. J Biol Chem 2006; 281: 21256-21265.

29. Mehta K, Fok J, Miller FR, Koul D, Sahin AA. Prognostic significance of tissue transglutaminase in drug resistant and metastatic breast cancer. Clin Cancer Res 2004; 10: 8068-8076.

30. Weston CR, Davis RJ. The JNK signal transduction pathway. Curr Opin Genet Dev 2002; 12: $14-21$

31. Tournier C, Hess $P$, Yang DD, Xu J, Turner TK, Nimnual A et al. Requirement of JNK for stress-induced activation of the cytochrome c-mediated death pathway. Science 2000; 288: 870-874.

32. Shin DM, Jeon JH, Kim CW, Cho SY, Kwon JC, Lee HJ et al. Cell type-specific activation of intracellular transglutaminase 2 by oxidative stress or ultraviolet irradiation: implications of transglutaminase 2 in age-related cataractogenesis. J Biol Chem 2004; 279: 15032-15039.

33. Fan G, Merritt SE, Kortenjann M, Shaw PE, Holzman LB. Dual leucine zipper-bearing kinase (DLK) activates p46SAPK and p38mapk but not ERK2. J Biol Chem 1996; 271: 24788-24793.

34. Gallo KA, Johnson GL. Mixed-lineage kinase control of JNK and p38 MAPK pathways. Nat Rev Mol Cell Biol 2002; 3: 663-672.

35. Johnson GV, Cox TM, Lockhart JP, Zinnerman MD, Miller ML, Powers RE. Transglutaminase activity is increased in Alzheimer's disease brain. Brain Res 1997; 751: 323-329.

36. Rasmussen LK, Sorensen ES, Petersen TE, Gliemann J, Jensen PH. Identification of glutamine and lysine residues in Alzheimer amyloid $\beta$ A4 peptide responsible for transglutaminase-catalysed homopolymerization and cross-linking to alpha $2 \mathrm{M}$ receptor. FEBS Lett 1994; 338: 161-166.

37. Tucholski J, Kuret J, Johnson GV. Tau is modified by tissue transglutaminase in situ: possible functional and metabolic effects of polyamination. $J$ Neurochem 1999; 73: 1871-1880.

38. Chen JSK, Konopleva M, Andreeff M, Multani AS, Pathak S, Mehta K. Drug-resistant breast carcinoma (MCF-7) cells are paradoxically sensitive to apoptosis. J Cell Physiol 2004: 200: 223-234.

39. Fok JY, Mehta K. Tissue transglutaminase induces the release of apoptosis inducing factor and results in apoptotic death of pancreatic cells. Apoptosis 2007; 12: 1455-1463.

40. Douziech M, Laberge G, Grondin G, Daigle N, Blouin R. Localization of the mixed-lineage kinase DLK/MUK/ZPK to the Golgi apparatus in NIH 3 T3 cells. J Histochem Cytochem 1999; 47: 1287-1296.

41. Zhang J, Lesort M, Guttmann RP, Johnson GVW. Modulation of the in situ activity of tissue transglutaminase by calcium and GTP. J Biol Chem 1998; 273: 2288-2295. 